I cc. cobalt chloride, $0.3 \mathrm{cc}$. ammonium hydroxide, ${ }^{1} 3 \mathrm{cc}$. of $95 \%$ alcohol, and water to make $50 \mathrm{cc}$.

Table VII shows that in $50 \mathrm{cc}$. of solution, $0.010 \mathrm{~g}$. of resorcinol can be detected in the presence of $0.200 \mathrm{~g}$. of hydroquinone.

Table VIII shows that in $50 \mathrm{cc}$ of solution, $0.005 \mathrm{~g}$. of resorcinol can be detected in the presence of $0.300 \mathrm{~g}$. of carbolic acid.

Table IX shows that in $50 \mathrm{cc}$. of solution, o.oro g. of resorcinol can be detected in presence of $0.200 \mathrm{~g}$. of pyrogallic acid.

\title{
Summary.
}

(I) Resorcinol in an ammoniacal solution of cobalt salts produces a color distinct from that produced by the other phenols tried.

(2) The best conditons for uniformity of this color development have been determined.

(3) The exclusion of the air from the test solution increases the length of time required for color development.

(4) The presence of certain other phenols either inhibit the formation of the resorcinol color, or else obscure it by the colors they themselves produce. Consequently, resorcinol cannot with certainty be detected with an ammoniacal solution of cobalt by the method indicated in Tables III to $\mathrm{V}$, if these other phenols are present.

(5) Resorcinol as low as $0.00 \mathrm{I}$ g. per $50 \mathrm{cc}$. sample, if shaken with a weak ammoniacal solution of a cobalt salt and a few cubic centimeters of alcohol, develops a dark green color. Pyrocatechin, hydroquinone, and pyrogallic acid under same conditions develop a brown color, carbolic acid produces a colorless solution.

(6) The presence of the other phenols tried does not prevent the formation of the dark green color developed by resorcinol. Thus small amounts of resorcinol can be detected in the presence of comparatively latge amounts of certain other phenols.

Madison, Wis.

[Contribution from the Carbohydrate laboratory, Bureau of Chemistry, [NITED States Department OF Agricliture.]

\section{PREPARATION OF BROMOACETYLGLUCOSE AND CERTAIN OTHER BROMOACETYL SUGARS.}

By J. K. DALE.

Received August 19, 1916.

In a recent article $\mathrm{E}^{2}$. Fischer published a method for the preparation of bromoacetylglucose which consisted essentially in the action of a saturated solution of hydrobromic acid gas in glacial acetic acid upon glucose pentacetate. Since bromoacetylglucose is an important compound, serving as the basis for the synthesis of many derivatives of glucose, it does not

1 In Table IX the amount of ammonium hydroxide was $2 \mathrm{cc}$. for each test solution.

${ }^{2}$ Ber.. 49, 584 (1916). 
seem out of place to describe a method I have used for its preparation which is somewhat simpler than that given by Fischer.

A saturated solution of hydrobromic acid gas in acetic anhydride was found to react directly with several of the sugars and from the reaction mixture could be isolated the bromoacetyl derivative. By this reaction bromoacetylxylose, ${ }^{1}$ bromoacetylcellulose and bromoacetyllactose, as well as bromoacetylglucose, were prepared in the crystalline condition with yields of respectively 26,60 and $77 \%$ of the theoretical. Bromoacetylmaltose was also obtained by this method, though only in the amorphous condition. A detailed description of the preparation of bromoacetylglucose is given below; it is typical of the method of preparation of each of the above named compounds.

Twenty-five grams of finely powdered glucose were treated in a large Erlenmeyer flask at room temperature with I25 cc. of acetic anhydride saturated with hydrobromic acid gas. A very vigorous reaction followed, leaving a clear, straw-colored sirup. This was cooled, mixed with 300 cc. of chloroform and the solution washed twice with water, once with enough sodium bicarbonate solution to neutralize the dissolved acids, and then once more with water. After drying with calcium chloride, the chloroform solution was evaporated at $50^{\circ}$ under diminished-pressure to a thick sirup which was washed into a beaker with a little dry ether. Fifteen to twenty volumes of petroleum ether were added, causing the bromoasetylglucose to precipitate as a thick sirup, which on cooling in an ice bath and being stirred vigorously, solidified in a few minutes to a crystalline mass. It was filtered off on a Büchner funnel and recrystallized by dissolving in $75 \mathrm{cc}$. of dry ether and evaporating the solution in a current of dry air until it crystallized. The yield of the once recrystallized substance was between $50 \%$ and $60 \%$ of the theory. Fischer obtained by his method $56 \%$ of the theoretical amount.

When bromoacetylglucose was sufficiently pure no trouble was experienced in preserving it in good condition for weeks at a time, but usually more than one recrystallization was necessary for this purpose.

WasRINGTON, D. C.

[From the INSTITUTE OF The Medical Chemistry of Kyoto Imperial UNIVERst'ty, JAPAN.]

QUANTITATIVE DETERMINATION OF HIPPURIC ACID IN URINE, BLOOD, MUSCLES AND LIVER: A METHOD.

By Dr. Hizo ITo.

Received July 28, 1916.

Since Bunge and Schmiedeberg ${ }^{2}$ published a well-known method for quantitative determination of hippuric acid in urine in 1876 , various

1 This Journal, 37, 2745 (I9i5).

2 Bunge u. Schmiedeberg, Arch. exp. Path. Pharm., 6, 233 (1876). 